\title{
BMJ Open Hypertension prevalence, incidence and risk factors among children and adolescents in Africa: a systematic review and meta-analysis protocol
}

\author{
Mickael Essouma, ${ }^{1}$ Jean Jacques N Noubiap, ${ }^{2,3}$ Jean Joel R Bigna, ${ }^{4}$ \\ Jobert Richie N Nansseu, ${ }^{5}$ Ahmadou M Jingi, ${ }^{6}$ Leopold N Aminde, ${ }^{7,8}$ Joseline Zafack ${ }^{9}$
}

To cite: Essouma $\mathrm{M}$, Noubiap JJN, Bigna JJR, et al. Hypertension prevalence, incidence and risk factors among children and adolescents in Africa: a systematic review and metaanalysis protocol. BMJ Open 2015;5:e008472.

doi:10.1136/bmjopen-2015008472

\section{- Prepublication history and additional material is available. To view please visit the journal (http://dx.doi.org/ 10.1136/bmjopen-2015- 008472).}

Received 11 April 2015 Revised 15 July 2015 Accepted 25 August 2015

For numbered affiliations see end of article.

Correspondence to Dr Jean Jacques N Noubiap; noubiapjj@yahoo.fr

\section{ABSTRACT}

Introduction: The African adult population is facing a growing epidemic of hypertension. Establishment of accurate epidemiological data on hypertension in African children and adolescents may have important implications for hypertension preventive strategies in Africa.

Methods and analysis: This systematic review and meta-analysis will follow the MOOSE Guidelines. Relevant abstracts published in English/French from 1 January 1985 to 31 July 2015 will be searched in PubMed, Google Scholar and Online African journals. Full texts of eligible studies will then be accessed through PubMed, Google Scholar, HINARI and the respective journals' websites. Relevant unpublished papers and conference proceedings will also be checked. Data will be analysed using R statistical software. The study-specific estimates will be pooled through a random-effects metaanalysis model to obtain an overall summary estimate of the prevalence/incidence of hypertension across studies. Also, we will assess the association between risk factors and hypertension. Heterogeneity of studies will be evaluated by the $\chi^{2}$ test on Cochrane's $Q$ statistic. Funnel plots analysis and Egger's test will be done to detect publication bias. Results will be presented by geographic region (central, eastern, northern, southern and western Africa). A $p$ value less than 0.05 will be considered significant for factors that predicted hypertension.

Ethics and dissemination: The current study is based on published data, and thus ethical approval is not required. This systematic review and meta-analysis is expected to serve as input for designing early life preventive and control strategies, and as a guide for future research based on existing gaps. The final report of the systematic review in the form of a scientific paper will be published in peer-reviewed journals. Findings will further be presented at conferences and submitted to relevant health authorities.

Trial registration number: CRD42015019029.

\section{INTRODUCTION}

Low-and-middle income countries (LMICs) are facing concurrent epidemics of communicable and non-communicable diseases. ${ }^{1}$ Unlike high-income countries, the prevalence of noncommunicable diseases has rapidly and continuously risen in the past decades in LMICs. ${ }^{2}$ This increasing burden of non-communicable diseases is particularly true for cardiovascular diseases (CVD), including stroke, coronary heart disease, peripheral arterial disease and heart failure. ${ }^{23}$ The high CVD-related morbidity and mortality observed in LMICs are paralleled by the increasing prevalence of lifestyle risk factors in these countries, especially hypertension. ${ }^{4}$ Hypertension, once rare in traditional African societies, ${ }^{5}{ }^{6}$ has become a major public health problem because of high prevalence rates contrasting with low awareness, treatment and control rates. ${ }^{7-9}$ The high prevalence of hypertension in Africa is due to both urbanisation and a shift towards western habits such as smoking, unhealthy diets with excess salt and fat intake, physical inactivity and consequential increased adiposity, and increasingly recognised non-traditional risk factors such as air pollution. ${ }^{5-7} 1011$

High blood pressure (BP) tracks from childhood to adulthood, such that children presenting with high BP (HBP) are at high risk of becoming hypertensive at an adult age. $^{5}{ }^{12-14}$ Emerging data from developed populations also suggest that, as in adults, primary hypertension is more common than secondary hypertension in the paediatric population. ${ }^{6}{ }^{15}$ Furthermore, the presence of HBP has been linked to end-organ damage (particularly left ventricular hypertrophy and kidney failure) and subclinical atherosclerosis. ${ }^{6} 1617$ Hence, in western countries, it is strongly recommended to measure BP in all children $\geq 3$ years of age in the course of routine healthcare, ${ }^{18}$ in order to promote cardiovascular health in adults.

It is difficult to estimate the prevalence of hypertension in the paediatric population 
because BP relates to sex, age and height during childhood, making it complicated to consider a single BP value as in adults. ${ }^{14} \quad 16 \quad 18 \quad 19$ Nevertheless, many epidemiological studies summarised in systematic reviews have attempted this issue in high-income countries, and reported an increasing burden of hypertension in children and adolescents, ${ }^{16}$ with prevalence rates of $1-5 \% .^{20}{ }^{21}$ This is not the case for African countries where there is a considerable variability of the estimated prevalence among studies conducted in South Africa and certain West African countries (0-22.3\%). ${ }^{5} 6192223$ Additionally, there is no reported incidence of hypertension among children in Africa, to the best of our knowledge, as well as limited evidence for its risk factors.

Thus, it is unclear whether the growing epidemic of hypertension in the African adult population is reflected in children and adolescents. The fast transition towards GVD in Africa, together with the increasing awareness of hypertension in children by African health professionals involved in paediatric care, stresses the need to build an accurate epidemiology of hypertension in children and adolescents in this part of the world. Along these lines, we present the protocol for a systematic review and meta-analysis to estimate the prevalence and incidence rates of hypertension among African children and adolescents, as well as its risk factors. Results are intended to provide an essential basis for designing cost-effective interventions that can be introduced early in life to prevent the burden of CVD in adulthood.

\section{OBJECTIVE}

To conduct a systematic review and meta-analysis to estimate the prevalence and the incidence of hypertension among children and adolescents in Africa, as well as its risk factors.

\section{Review question}

This review of studies published in the past 30 years, from 1 January 1985 to 30 April 2015, should answer the following questions:

1. What is the prevalence of HBP among African children and adolescents residing in Africa?

2. What is the incidence of HBP among African children and adolescents?

3. Which factors are associated with HBP among African children and adolescents?

\section{Criteria for considering studies for the review Inclusion criteria}

We will include:

1. Cross-sectional, case-control or cohort studies of children and adolescents aged 1-18 years residing in African countries reporting the prevalence or incidence of HBP, or enough data to compute these estimates.

2. Studies including assessment of office and/or ambulatory and/or home $\mathrm{BP}$, as well as those evaluating risk factors for hypertension in African children and adolescents.

3. Studies with a diagnosis of hypertension based on BP $\geq 95$ th centile.

4. Studies published in English or French, unpublished studies and conference proceedings.

\section{Exclusion criteria}

1. Studies on non-systemic hypertension (intracranial hypertension, pulmonary hypertension).

2. Studies conducted among populations of African origin residing outside of Africa.

3. Studies in subgroups of participants selected on the basis of the presence of hypertension (eg, clinical trials).

4. Studies including adult and paediatric populations in which it will not be possible to extract data of children and/or adolescents.

5. Case series with small sample size (less than 50 participants), letters, reviews, commentaries and editorials.

6. Studies lacking primary data and/or explicit method description.

7. Duplicates: for studies published in more than one paper, the most comprehensive one reporting the largest sample size will be considered.

8. Studies with serious ethical issues.

9. Studies whose full data will not be accessible even after request from the authors.

\section{Search strategy for identifying relevant studies}

This systematic review and meta-analysis will follow the guidelines set out by MOOSE. ${ }^{24}$ The search strategy will be implemented in two stages:

\section{Bibliographic database searches}

A. Relevant abstracts published in English or French on the prevalence, the incidence and the risk factors for HBP in children and adolescents in Africa will be identified via searching PubMed, Google Scholar and online African journals. The search will be limited to studies published between 1 January 1985 and 31 July 2015. Both text words and medical subject heading terms will be used. Key search terms will be: Africa, children, adolescents, prevalence, incidence, risk factors and hypertension. We will also use individual country names for the 53 African countries and hypertension as additional key search terms for more abstracts on the subject. Conference proceedings of the study period will also be identified through Medline and checked. The main search strategy is shown in table 1.

B. The abstracts of all eligible papers will be reviewed and full articles will be accessed through PubMed, Google Scholar, HINARI or journals' websites. The references of all the relevant research articles will also be scrutinised for additional potential data sources, and their full texts will be accessed in a similar way. The authors whose full text papers will 


\begin{tabular}{|c|c|}
\hline Search & Search terms \\
\hline 1 & Hypertension [tw] OR high blood pressure [tw] OR systolic hypertension [tw] OR diastolic hypertension [tw] \\
\hline 2 & Hypertension [MeSH terms] \\
\hline 3 & \# 1 OR \# 2 \\
\hline 4 & $\begin{array}{l}\text { (Child [tw] OR Child [MeSH terms] Children [tw] OR childhood [tw] OR adolescent [MeSH terms] OR adolescent } \\
\text { [tw] OR adolescent* [tw] OR pediatric [tw] OR teens [tw] OR teen [tw] OR Teenage* [tw] OR Youth* [tw] OR Infan* } \\
\text { [tw]) }\end{array}$ \\
\hline 5 & \# 3 AND \# 4 \\
\hline 6 & $\begin{array}{l}\text { ((((“Africa"[MeSH] OR Africa* [tw] OR Algeria[tw] OR Angola[tw] OR Benin[tw] OR Botswana[tw] OR "Burkina } \\
\text { Faso"[tw] OR Burundi [tw] OR Cameroon [tw] OR "Canary Islands" [tw] OR "Cape Verde" [tw] OR "Central African } \\
\text { Republic" [tw] OR Chad [tw] OR Comoros [tw] OR Congo [tw] OR "Democratic Republic of Congo" [tw] OR Djibouti } \\
\text { [tw] OR Egypt [tw] OR "Equatorial Guinea" [tw] OR Eritrea [tw] OR Ethiopia [tw] OR Gabon [tw] OR Gambia [tw] OR } \\
\text { Ghana [tw] OR Guinea [tw] OR "Guinea Bissau" [tw] OR "Ivory Coast" [tw] OR "Cote d'Ivoire" [tw] OR Jamahiriya } \\
\text { [tw] OR Jamahiryia [tw] OR Kenya [tw] OR Lesotho [tw] OR Liberia [tw] OR Libya [tw] OR Libia [tw] OR } \\
\text { Madagascar [tw] OR Malawi [tw] OR Mali [tw] OR Mauritania [tw] OR Mauritius [tw] OR Morocco [tw] OR } \\
\text { Mozambique [tw] OR Mocambique [tw] OR Namibia [tw] OR Niger [tw] OR Nigeria [tw] OR Principe [tw] OR } \\
\text { Reunion [tw] OR Rwanda [tw] OR "Sao Tome" [tw] OR Senegal [tw] OR Seychelles [tw] OR "Sierra Leone" [tw] OR } \\
\text { Somalia [tw] OR "South Africa" [tw] OR "St Helena" [tw] OR Sudan [tw] OR Swaziland [tw] OR Tanzania [tw] OR } \\
\text { Togo [tw] OR Tunisia [tw] OR Uganda [tw] OR "Western Sahara" [tw] OR Zaire [tw] OR Zambia [tw] OR Zimbabwe } \\
\text { [tw] OR "Central Africa" [tw] OR "Central African" [tw] OR "West Africa" [tw] OR "West African" [tw] OR "Western } \\
\text { Africa" [tw] OR "Western African" [tw] OR "East Africa" [tw] OR "East African" [tw] OR "Eastern Africa" [tw] OR } \\
\text { "Eastern African" [tw] OR "North Africa" [tw] OR "North African" [tw] OR "Northern Africa" [tw] OR "Northern African" } \\
\text { [tw] OR "South African" [tw] OR "Southern Africa" [tw] OR "Southern African" [tw] OR "sub Saharan Africa" [tw] OR } \\
\text { "sub Saharan African" [tw] OR "subSaharan Africa" [tw] OR "subSaharan African" [tw]) NOT ("guinea pig" [tw] OR } \\
\text { "guinea pigs" [tw] OR "aspergillus niger" [tw]))))) }\end{array}$ \\
\hline 7 & \# 5 AND \# 6 \\
\hline 8 & \#7 Limits: $1985 / 01 / 01$ to $2015 / 04 / 30$ and studies done in Humans, in English and French \\
\hline
\end{tabular}

not be accessible by the numerous internet-based sources will be directly contacted to provide them. In case of no feedback from these authors, the corresponding studies will be excluded.

\section{Selection of studies for inclusion in the review}

Assessment of eligible papers will be done by three members of the team independently, and using an assessment guide to ensure that the selection criteria are reliably applied by all the investigators. They will also consensually retain the studies that will be included in the review, and any disagreement will be solved by a fourth assessor.

\section{Assessment of methodological quality and data reporting}

The Risk of Bias Tool for Prevalence Studies developed by Hoy et $a l^{25}$ (see online supplementary material appendix S1), and the Cochrane guidelines available in Review Manager V.5.3 (http://tech.cochrane.org/revman) will be used to assess the methodological quality and risk of bias for each study. The STROBE checklist (see online supplementary material appendix S2) ${ }^{26}$ will be used to evaluate the reporting methodology in each paper. Risk of bias and quality scores will be presented in a table.

\section{Data extraction and management}

A data extraction sheet will be used to collect information about the country, the year of publication, the language of publication, the type of publication, the study design, the number of participants, the mean age of the population, the diagnostic criteria for hypertension, the prevalence and the incidence of hypertension, as well as its predictive factors when available. Where prevalence/incidence rates or information for calculating them (eg, sample size, number of outcomes) are lacking, we will directly contact the corresponding author to request the information. In case of multinational studies, we will separate the results to show the prevalence, the incidence and risk factors within individual countries. Where it will not be possible to disaggregate the data by country, the study will be presented as one and the countries in which the study was done will be shown.

\section{Statistical analysis}

Data will be analysed using Stata software (Stata Corp V.13, Texas, USA). A meta-analysis will be conducted for data obtained from studies in which hypertension was defined identically. SEs for the study-specific estimates will first be determined from the point estimate and the appropriate denominators, assuming a binominal (or Poisson for incidence data) distribution. Then the study-specific estimates will be pooled through a random-effects meta-analysis model, to obtain an overall summary estimate of the prevalence/incidence across studies, after stabilising the variance of individual studies using the Freeman-Tukey double arc-sine transformation. ${ }^{27} \mathrm{~A}$ meta-analysis will be 
performed to assess the association between risk factors and hypertension.

Heterogeneity will be evaluated by the $\chi^{2}$ test on Cochrane ${ }^{28}$ s $Q$ statistic, which is quantified by $\mathrm{I}^{2}$ values ${ }^{29}$ assuming that $\mathrm{I}^{2}$ values of $25 \%, 50 \%$ and $75 \%$, respectively, represent low, medium and high heterogeneity. Where substantial heterogeneity will be detected, a subgroup analysis will be performed to detect its possible sources using the following grouping variables: age group, sex, study setting (hospital vs community-based), geographical area (central, eastern, northern, southern and western Africa), study quality. Inter-rater agreement for study inclusion will be assessed using Cohen's $\kappa$ coefficient. ${ }^{30}$ Funnel plots analysis and Egger's test ${ }^{31}$ will be done to detect publication bias.

Results will be presented by geographic region (central, eastern, northern, southern and western Africa). A p value less than 0.05 will be considered significant for factors that predicted hypertension.

\section{Results reporting and presentation}

The study selection process will be summarised using a flow diagram. Reasons for studies' exclusion will be described. This will follow the MOOSE Guidelines for Meta-Analyses and Systematic Reviews of Observational Studies. ${ }^{24}$ Quantitative data will be presented in evidence tables of individual studies as well as in summary tables and funnel plots where appropriate. We will examine prevalence/incidence and risk factors by region, setting (hospital or community), time period and disease-specific populations depending on the data available. We plan to report on quality scores and risk of bias for each eligible study. This may be tabulated and accompanied by narrative summaries.

\section{Conclusion}

Hypertension, a major driver of the CVD burden in Africa, has reached epidemic proportions in the adult population. Given that children and adolescents presenting with HBP have a major risk of becoming hypertensive adults, specific cost-effective interventions need to be introduced early in life to prevent CVD in adulthood. Prior to these strategies, accurate epidemiological data should be obtained. We wish that this review will guide policy, practice and research by providing information on the magnitude of hypertension among African children, as well as its risk factors, and the remaining gaps that may form the basis for future studies.

A major possible limitation of this study could be the limited data with predominance of hospital-based studies and poor quality data when available. Another possible limitation may be the heterogeneity of studies, making further analysis difficult. In addition, there may be a predominance of cross-sectional studies, making it difficult to obtain reliable incidence estimates and to determine risk factors for hypertension. Other drawbacks might include the non-random selection of participants, and the under-representation of some geographical areas such as Central Africa. These problems have already been noted by previous reviews on non-communicable diseases in Africa, ${ }^{1-3} 832$ and might be reinforced by the fact that BP is still not routinely recorded in paediatric consultations in many African countries. Finally, since we will include only studies published in English or French, we may lose relevant data from countries where these languages are not spoken.

\section{ETHICS AND DISSEMINATION}

This study is based on published data, and therefore ethical approval is not a requirement. This systematic review and meta-analysis is expected to serve as a basis for designing preventive and control strategies early in life, and as a guide for future research based on the remaining gaps. The final report of this study in the form of a scientific paper will be published in peer-reviewed journals. Findings will further be presented at conferences and submitted to relevant health authorities. We also plan to update the review in the future to monitor changes and guide health service and policy solutions.

\section{Author affiliations}

${ }^{1}$ Division of Medicine, Sangmelima Referral Hospital, Sangmelima, Cameroon

${ }^{2}$ Department of Medicine, Groote Schuur Hospital and University of Cape Town, Cape Town, South Africa

${ }^{3}$ Medical Diagnostic Center, Yaoundé, Cameroon

${ }^{4}$ Department of Epidemiology and Public Health, Pasteur Center of Cameroon, Yaoundé, Cameroon

${ }^{5}$ Department of Public Health, Faculty of Medicine and Biomedical Sciences, Yaoundé, Cameroon

${ }^{6}$ Department of Internal Medicine and Specialties, Faculty of Medicine and Biomedical Sciences, University of Yaoundé I, Yaoundé, Cameroon

${ }^{7}$ Nguti District Hospital, Nguti, Cameroon

${ }^{8}$ Clinical Research Education, Networking and Consultancy (CRENC), Douala, Cameroon

${ }^{9}$ Department of Preventive and Social Medicine, Laval University, Québec City, Québec, Canada

Contributors JJNN, ME and JJRB conceived and designed the protocol, and ME drafted the manuscript. JJNN, JJRB, JRNN, AMJ, LNA and JZ critically revised the manuscript for methodological and intellectual content. JJNN is the guarantor of the review. All authors approved the final version.

\section{Competing interests None declared.}

Provenance and peer review Not commissioned; externally peer reviewed.

Open Access This is an Open Access article distributed in accordance with the Creative Commons Attribution Non Commercial (CC BY-NC 4.0) license, which permits others to distribute, remix, adapt, build upon this work noncommercially, and license their derivative works on different terms, provided the original work is properly cited and the use is non-commercial. See: http:// creativecommons.org/licenses/by-nc/4.0/

\section{REFERENCES}

1. GBD 2013 Mortality and Causes of Death Collaborators. Global, regional, and national age-sex specific all-cause and cause-specific mortality for 240 causes of death, 1990-2013: a systematic analysis for the Global Burden of Disease Study 2013. Lancet 2015;385:117-71.

2. Joshi R, Alim M, Kengne AP, et al. Task shifting for non-communicable disease management in low and middle income countries--a systematic review. PLoS ONE 2014;9:e103754.

3. Adeloye $D$. An estimate of the incidence and prevalence of stroke in Africa: a systematic review and meta-analysis. PLOS ONE 2014;9: e100724. 
4. Lim SS, Vos T, Flaxman AD, et al. A comparative risk assessment of burden of disease and injury attributable to 67 risk factors and risk factor clusters in 21 regions, 1990-2010: a systematic analysis for the Global Burden of Disease Study 2010. Lancet 2012;380:2224-60.

5. Ejike CECC, Ugwu CE, Ezeanyika LU, et al. Blood pressure patterns in relation to geographic area of residence: a crosssectional study of adolescents in Kogi state, Nigeria. BMC Public Health 2008;8:411.

6. Agyemang C, Redekop WK, Owusu-Dabo E, et al. Blood pressure patterns in rural, semi-urban and urban children in the Ashanti region of Ghana, West Africa. BMC Public Health 2005;5:114.

7. Iwelunmor J, Airhihenbuwa CO, Cooper R, et al. Prevalence, determinants and systems-thinking approaches to optimal hypertension control in West Africa. Glob Health 2014;10:42.

8. Kayima J, Wanyenze RK, Katamba A, et al. Hypertension awareness, treatment and control in Africa: a systematic review. BMC Cardiovasc Disord 2013;13:54.

9. Kingue S, Ngoe CN, Menanga AP, et al. Prevalence and correlates of hypertension in Cameroon: a nationwide population-based cross-sectional study. J Clin Hypertens (Greenwich) 2015. doi:10.1111/jch.12604 [epub ahead of print 3 Jul 2015].

10. Noubiap JJ, Bigna JJ, Nansseu JR. Low sodium and high potassium intake for cardiovascular prevention: evidence revisited with emphasis on challenges in sub-Saharan Africa. J Clin Hypertens Greenwich Conn 2015;17:81-3.

11. Noubiap JJ, Essouma M, Bigna JJ. Targeting household air pollution for curbing the cardiovascular disease burden: a health priority in Sub-Saharan Africa. J Clin Hypertens (Greenwich) 2015. doi:10. 1111/jch.12610 [epub ahead of print 3 Jul 2015]

12. Ferrer M, Fernández-Britto JE, Bacallao J, et al. Development of hypertension in a cohort of cuban adolescents. MEDICC Rev 2015;17:41-7.

13. Williams CL, Hayman LL, Daniels SR, et al. Cardiovascular health in childhood: a statement for health professionals from the Committee on Atherosclerosis, Hypertension, and Obesity in the Young (AHOY) of the Council on Cardiovascular Disease in the Young, American Heart Association. Circulation 2002;106:143-60.

14. Lurbe E, Cifkova R, Cruickshank JK, et al, Sociedad Europea de Hipertensión. [Management of high blood pressure in children and adolescents: recommendations of the European Society of hypertension]. An Pediatría Barc Spain 2010;73:51.e1-28.

15. Sorof JM, Lai D, Turner J, et al. Overweight, ethnicity, and the prevalence of hypertension in school-aged children. Pediatrics 2004;113(3 Pt 1):475-82.

16. Feber J, Ahmed M. Hypertension in children: new trends and challenges. Clin Sci Lond Engl 2010;119:151-61.
17. Hanevold C, Waller J, Daniels S, et al., International Pediatric Hypertension Association. The effects of obesity, gender, and ethnic group on left ventricular hypertrophy and geometry in hypertensive children: a collaborative study of the International Pediatric Hypertension Association. Pediatrics 2004;113:328-33.

18. Falkner B, Daniels SR. Summary of the fourth report on the diagnosis, evaluation, and treatment of high blood pressure in children and adolescents. Hypertension 2004;44:387-8.

19. Chiolero A, Madeleine G, Gabriel A, et al. Prevalence of elevated blood pressure and association with overweight in children of a rapidly developing country. J Hum Hypertens 2007;21:120-7.

20. Redwine KM, Acosta AA, Poffenbarger T, et al. Development of hypertension in adolescents with pre-hypertension. $J$ Pediatr 2012;160:98-103.

21. Kollias A, Dafni M, Poulidakis E, et al. Out-of-office blood pressure and target organ damage in children and adolescents: a systematic review and meta-analysis. J Hypertens 2014;32:2315-31; discussion 2331.

22. Monyeki $\mathrm{K}$, Kemper $\mathrm{H}$. The risk factors for elevated blood pressure and how to address cardiovascular risk factors: a review in paediatric populations. J Hum Hypertens 2008;22:450-9.

23. Woelk G, Emanuel I, Weiss NS, et al. Birthweight and blood pressure among children in Harare, Zimbabwe. Arch Dis Child Fetal Neonatal Ed 1998;79:F119-22.

24. Stroup DF, Berlin JA, Morton SC, et al. Meta-analysis of observational studies in epidemiology: a proposal for reporting. Meta-analysis Of Observational Studies in Epidemiology (MOOSE) group. JAMA 2000;283:2008-12.

25. Hoy $D$, Brooks $P$, Woolf $A$, et al. Assessing risk of bias in prevalence studies: modification of an existing tool and evidence of interrater agreement. J Clin Epidemiol 2012;65:934-9.

26. Von Elm E, Altman DG, Egger M, et al. The Strengthening the Reporting of Observational Studies in Epidemiology (STROBE) statement: guidelines for reporting observational studies. Prev Med 2007:45:247-51.

27. Barendregt JJ, Doi SA, Lee YY, et al. Meta-analysis of prevalence. $J$ Epidemiol Community Health 2013;67:974-8.

28. Cochran $\mathrm{W}$. The combination of estimates from different experiments. Biometrics 1954;10:101-29.

29. Higgins JP, Thompson SG. Quantifying heterogeneity in a metaanalysis. Stat Med 2002;21:1539-58.

30. Landis JR, Koch GG. The measurement of observer agreement for categorical data. Biometrics 1977;33:159-74.

31. Egger M, Davey Smith G, Schneider M, et al. Bias in meta-analysis detected by a simple, graphical test. BMJ 1997;315:629-34.

32. Noubiap JJ, Naidoo J, Kengne AP. Diabetic nephropathy in Africa: a systematic review. World J Diabetes 2015;6:759-73. 